Article

\title{
Evaporation Induced Crystal Nucleation and Morphology of Dried Poly (Vinylidene Fluoride) Droplets
}

\author{
Yongri Liang ${ }^{1,2 *}$, and Susu Wang ${ }^{2}$
}

1 State Key Lab of Metastable Materials Science and Technology, and School of Materials Science and Engineering, Yanshan University, No. 438 West Hebei Avenue, Qinhuangdao, Hebei 066004, P.R. China; liangyr@ysu.edu.cn

2 College of Materials Science and Engineering, Beijing Institute of Petrochemical Technology, Beijing 102617, P. R. China; wangsusu@bjfleming.com

* Correspondence: liangyr@ysu.edu.cn ; Tel.: (+86-18010156705)

\begin{abstract}
The evaporation of polymer solution droplet is an important in solution-based polymer film fabrications, such as inkjet print, spray coatings, and droplet casting etc. In this work, we investigated the effect of droplet size, solvent evaporation rate, and concentration on "coffee-ring" effect, crystal nucleation, polymorphism, and morphology of dried poly (vinylidene fluoride) (PVDF) solution droplets with atomic force microscopy (AFM) method. We found that the crystal structure, morphology and crystal distribution in the center and edge regions of dried PVDF droplets were different due to "coffee-ring" effect. The "coffee-ring" effect of dried PVDF droplets was mainly composited of accumulated crystals at the edge region of droplet, which was mainly made by crystallization of migrated chains. The interplay between migration of chains, crystallization and solidification of PVDF droplets significantly influenced the formation of "coffee-ring". In addition, our results showed that the decrease of droplet size and controlling solvent evaporation rate were effective ways to improve the PVDF $\beta$-phase nucleation and decrease crystal size.
\end{abstract}

Keywords: Evaporation; poly (vinylidene fluoride); Crystallization; Droplet; Coffee ring effect; Ultrasonic spray

\section{Introduction}

The evaporation of polymer solution droplets is very important in some solution processes-based polymer film fabrications, such as inkjet print, spray coatings, and droplet casting etc.[1] Evaporation process of polymer solution droplets is very complex nonequilibrium process, where many factors influence the final morphology and structure of dried polymer solution droplets, such as evaporation rate, diffusion rate of solute, and phase transition etc.[2] In contrast with solution casting and blade coating processes, the evaporation of droplets generally generates "coffee ring" effect which is generally occurred by evaporation induced outward capillary flow. [3-7] Therefore, understanding the interplay between solvent evaporation and crystallization of polymer solution droplets is very important to control the final morphology of dried polymer solution droplets and droplet deposited polymer films.

In the droplet evaporation, the capillary outward flow will be induced inside the pinned droplet, and can carry almost all the dispersed solute to the edge to form a ringlike deposit. I.e., pinning of the drop at triple-phase contact lines (TPCL) along with faster evaporation leads to the defined ring structure. In addition, a concentration driven surface tension gradient leading to Marangoni flow that drives the solution in a path opposite to the outward capillary flow.[3] So, there is a competition between capillary convection and solute Marangoni flow and only a weak Marangoni flow of the solution can create a "coffee ring". Recently, Hu et al.[8] investigated the "spherulites ring" morphology and "coffee ring" profile of polyethylene oxide (PEO) film formed by the drying droplet at glass 
substrate with different heating rate. They found that the outward capillary flow is the dominant micro-flow inside drying droplet of $\mathrm{PEO}$ aqueous solution when substrate temperature is below $50{ }^{\circ} \mathrm{C}$. The solute is carried by the capillary flow to the edge of the droplet, and forms the profile of "coffee-ring". The spherulites get together in the center of PEO film. Marangoni convection carries much solute to the middle of droplets when substrate temperature is above $70{ }^{\circ} \mathrm{C}$, and forms the regular "spherulites ring" at the position of Marangoni convection center. Rietveld et al.[9] investigated the effect of electrospray process on the morphology of PVDF films. They suggested that the droplet size of the spray and the viscosity of the solution at deposition are two main factors to determine the morphology of PVDF film, and the solvent is the key parameter to produce polymer thin films in the electrospray process. More recently, we found that high $\beta$-phase content and high transparency of poly (vinylidene fluoride) (PVDF) film can be achieved by ultrasonic spray coating (USC) mothed. [10] In contrast with conventional blade coating process, the evaporation induced outward capillary flow can play an important role in the nucleation of PVDF $\beta$-phase in the USC process due to fast solvent evaporation. However, as far as we know, the evaporation induced crystal nucleation and morphology of polymer solution droplets has not been fully understood so far.

In this work, we prepared various size of PVDF solution droplets by dropping and USC methods, and investigated the effect of droplet size, solvent evaporation rate and concentration on the crystal nucleation and morphology of dried PVDF droplets with atomic force microscopy (AFM), and also investigated the crystal structure and morphology of ultrasonic spray coated PVDF films.

\section{Materials and Methods}

PVDF $(M w \approx 534,000)$ was purchased from Sigma-Aldrich Co. China. The Indium tin oxide (ITO) glasses with $6 \Omega$ of resistivity were purchased from Beijing Zhenyongwei Technology Co., Ltd. The ethyl alcohol, acetone (AC), and N, N- dimethylformamide (DMF) were purchased from Beijing Chemical Works. And all the chemicals were analytical reagent, and were used without further purification.

The PVDF powders were dissolved in a selected solvent at $70^{\circ} \mathrm{C}$ for 4 hours for preparation of the PVDF solutions with $12 \mathrm{mg} / \mathrm{ml}$ of concentration. The ITO glasses were used as a substrate for drying the PVDF solution droplets. The ITO glasses were cleaned in deionized water and ethyl alcohol for $5 \mathrm{~min}$ in ultrasonic bath in turn. The washed ITO glasses were dried by nitrogen gas, and then were treated by plasma for 3 minutes before use. A few millimeters diameter of PVDF solution large size (L) droplets were prepared by dropping $2 \mu \mathrm{l}$ of PVDF solution using $5 \mathrm{ml}$ of syringe, and dried at room temperature. The tens micrometers diameter of PVDF solution small size (S) droplets were prepared by ultrasonic spray method[10] at room temperature or $70{ }^{\circ} \mathrm{C}$. The height of ultrasonic nozzle (Siansonic Technology Co.,Ltd., China) from substrate was $15 \mathrm{~cm}$ for ultrasonic spray coating with $1 \mathrm{~W}$ of the power.

The ultrasonic spray coated PVDF films were prepared using $12 \mathrm{mg} / \mathrm{ml}$ of concentration with mixture solvent of DMA/AC (6/4 v/v). The ITO-coated glasses were used as a substrate for coating PVDF films. The ITO-coated glasses were cleaned in deionized water and ethyl alcohol for 5 min in ultrasonic bath in turn, and then were dried by nitrogen gas, and then were treated by plasma for 3 minutes before use. The height of ultrasonic nozzle from substrate was $15 \mathrm{~cm}$ and $0.1 \mathrm{ml} / \mathrm{min}$ of flow rate for ultrasonic spray coating with $1 \mathrm{~W}$ of the power.

The morphology of dried PVDF films were characterized by atomic force microscopy (AFM) in MFP-3D Origin AFM system (Oxford Instruments Co. USA), and optical microscopy (OM) (LV100ND, Nikon co. Ltd., Japan). The AFM tips (AC240TS-R3, Asylum Research) with $2 \mathrm{~N} / \mathrm{m}$ spring constant and $70 \mathrm{kHz}$ of resonance frequency were used to measurements. The Fourier transform infrared spectroscopy (FTIR) measurements are carried out in FTIR-850 Fourier transform infrared spectrometer (TIANJIN GANGDONG Co., China) with transmission mode using $4 \mathrm{~cm}-1$ of resolution and 32 times of scan. 


\section{Results}

\subsection{Solvent evaporation induced crystal nucleation and morphology of PVDF/DMF solution large size droplet}

To investigate the effect of droplet size on PVDF nucleation and morphology, we prepared L-and S- sizes of PVDF/DMF solution drops with DMF solvent. Figure 1 shows $\mathrm{OM}$ and AFM image of partial dried PVDF/DMF solution L- droplet, respectively. The OM image of dried PVDF/DMF solution L- droplet showed about $5.03 \mathrm{~mm}$ of diameter. And, the crystals presented in edge region of PVDF L-droplet is denser than that in center region. It indicates that evaporation of L-droplet can form "coffee ring" effect. The "coffee ring" effect is generally occurred at triple-phase contact lines (TPCL) in the case of evaporation of droplets due to outward capillary flow[3,5].

(A)

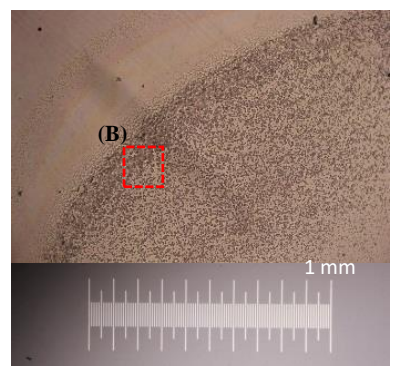

Diameter of dried droplet: $5.03 \mathrm{~mm}$

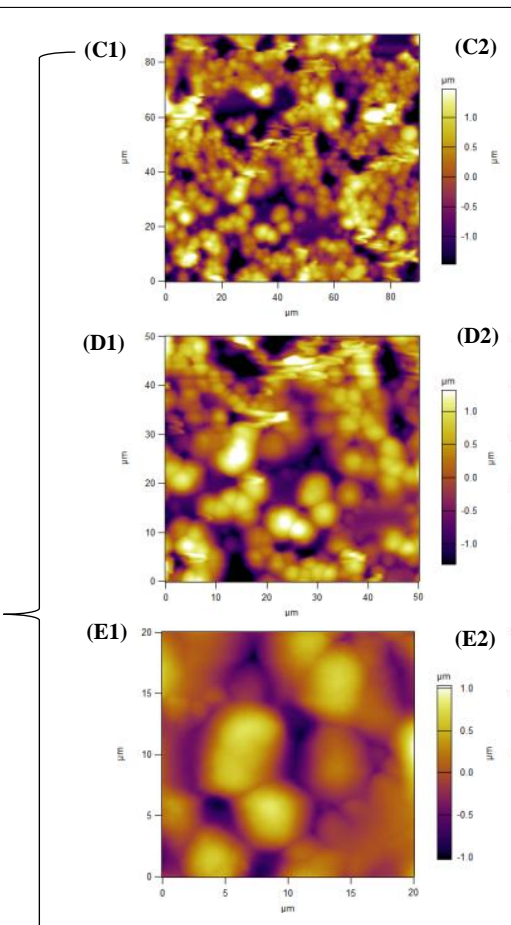

$$
\text { (F1) }
$$

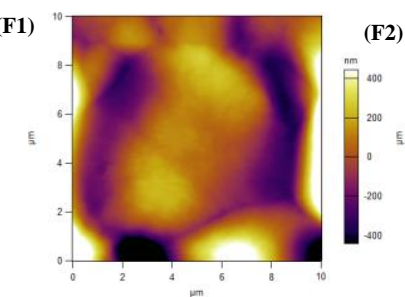

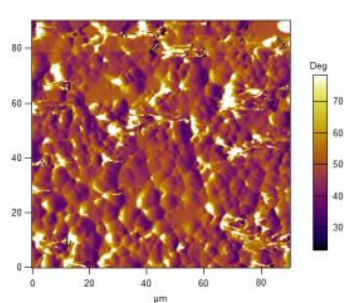

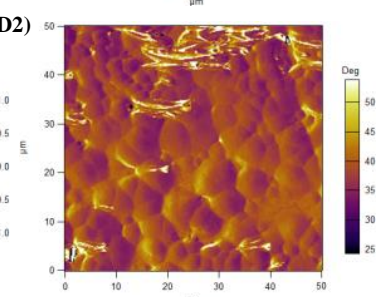

E2)
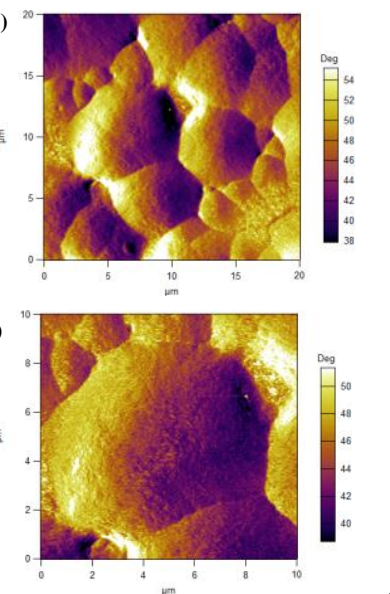

Figure 1. (A) Optical microscopy image of partial dried PVDF/DMF solution droplet with about $5.03 \mathrm{~mm}$ of diameter. (B) AFM images of edge region of dried PVDF/DMF solution droplet. (C-F) AFM height and phase images dried PVDF/DMF droplet. (1) height image, (2) phase images, (A) $90 \mu \mathrm{m} \times 90 \mu \mathrm{m}$; (B) $50 \mu \mathrm{m} \times 50 \mu \mathrm{m}$; (C) $20 \mu \mathrm{m} \times 20$ $\mu \mathrm{m}$; (D) $10 \mu \mathrm{m} \times 10 \mu \mathrm{m}$.

The crystals in the edge region of dried PVDF/DMF solution L-droplet showed about few micrometers size of particle like crystals which has hill-like shaped morphology and smooth surface (Figure $1 \mathrm{~F}$ ), and showed accumulated each other. (Figure $1 \mathrm{C}$ and D). Whereas, the crystals in the center region of dried PVDF/DMF solution L-droplet are exhibited a jigsaw puzzle like pieced together having remarkable boundaries (Figure 2). It means that the PVDF crystals in the center region are collided after crystal growth, which is different with the situation of accumulated crystals in the edge region. I.e., the mechanism of crystal nucleation and growth of PVDF in center region is different with that in edge region. Since the evaporation rate in edge region is faster than that in center region, 
the crystal nucleation and growth in edge region should be more constrained by fast evaporation caused solidification. In addition, the evaporation induced outward capillary flow can influence the accumulation of polymer coils and crystals. For the crystal accumulation in the dried L-droplet edge region, two possible mechanisms may be possible. One possibility is that the crystal nuclei are formed at edge region, and the crystal growth is limited by fast evaporation induced solidification. In that case, the polymer chains may be migrated from center region to edge region of droplet by evaporation induced outward capillary flow before crystallization. The other one possibility is that the crystal nuclei are formed at a certain center region, and then the small size of crystals are migrated to edge region to be accumulated. As shown in Figure (C)-(E), it seems to a few crystals randomly meet to be assembled. Therefore, we cannot rule out that the formed at center region of crystals are migrated to the edge of droplet to form "coffee-ring".

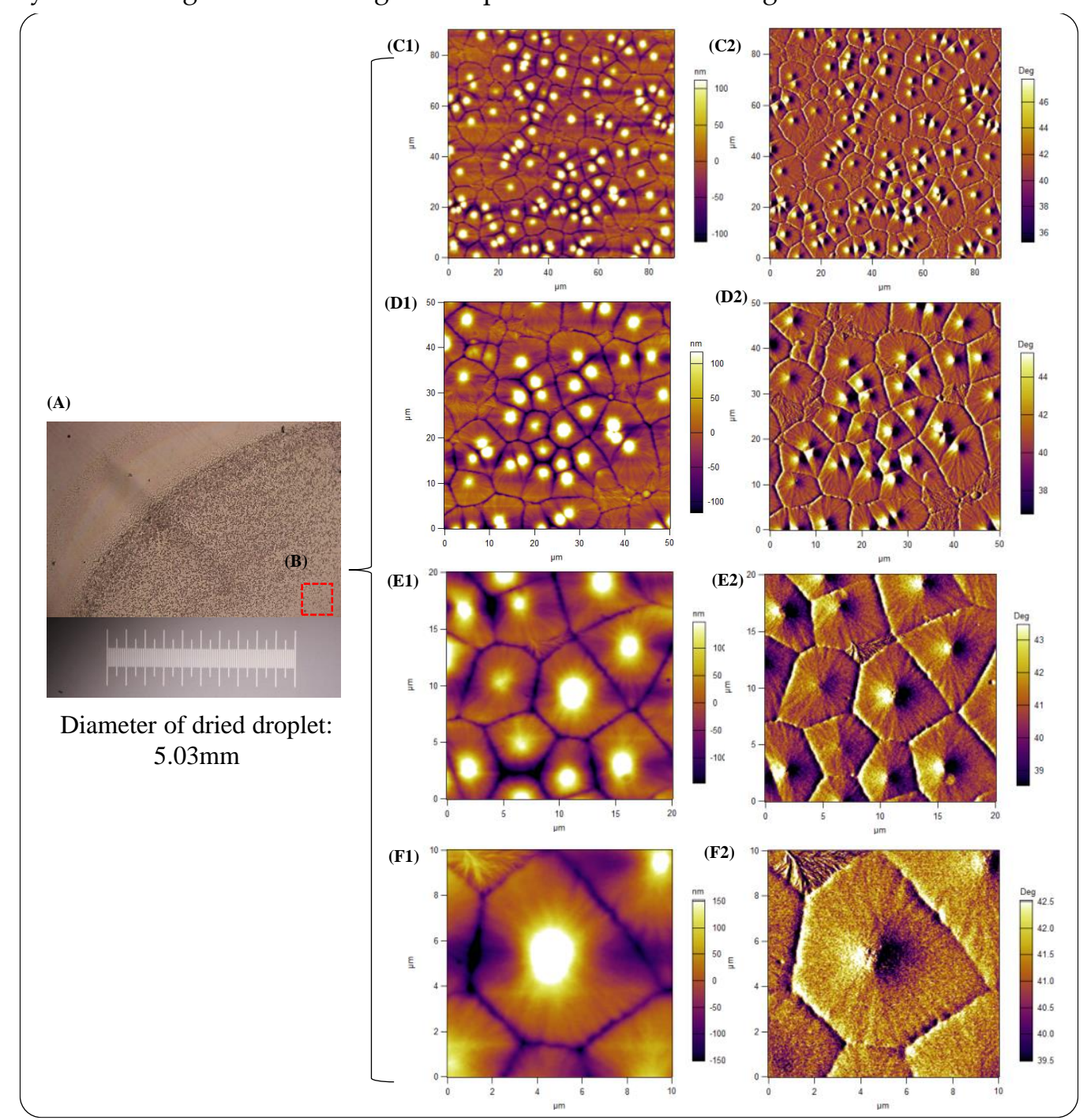

Figure 2. (A) Optical microscopy image of dried PVDF/DMF solution droplet with about $5.03 \mathrm{~mm}$ of diameter. (B) AFM images of center region of dried PVDF/DMF solution droplet. (C-F) AFM height and phase images dried PVDF/DMF solution droplet. (1) height image, (2) phase images, (A) $90 \mu \mathrm{m} \times 90 \mu \mathrm{m}$; (B) $50 \mu \mathrm{m} \times 50 \mu \mathrm{m}$; (C) $20 \mu \mathrm{m} \times 20$ $\mu \mathrm{m}$; (D) $10 \mu \mathrm{m} \times 10 \mu \mathrm{m}$.

In addition, the size and shape of PVDF crystals in the edge region are completely different with that in center region. Th crystals in edge region are showed about few micrometers size of polygon shaped spherulites which has Mongolian yurt roof like morphology with striped surface as shown in Figure 1 (C-F). And, we found that dendritic crystals are filled in the gaps of some spherulites boundaries in the center region of droplet 
as shown in Figure $2(\mathrm{E}-\mathrm{F})$ and Figure 3. The various crystal morphology of PVDF is caused by various crystalline phase. The hill-like shaped crystals is assigned to the $\beta$-form crystal of PVDF[11] and the Mongolian yurt roof shaped crystals may be assigned to the mixture of $\beta$-form or $\gamma$-phase crystals. [12] The dendritic-like crystals are assigned to the $\alpha$-form crystal of PVDF. [11] It means that the nucleation of dendritic-like $\alpha$-form crystals are developed at edge of spherulites and then are growth to filling in the gaps of interspherulites.

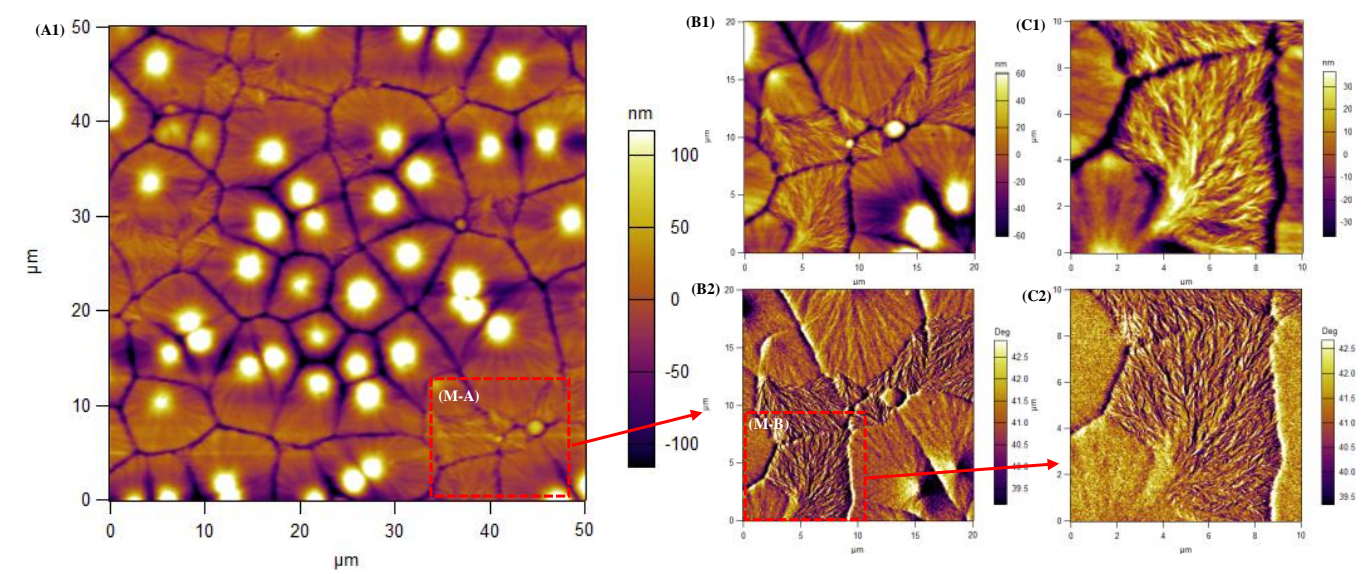

Figure 3. AFM height and phase images of center region of dried PVDF/DMF solution Ldroplet. (1) height image, (2) phase image, (A) $50 \mu \mathrm{m} \times 50 \mu \mathrm{m},(B) 20 \mu \mathrm{m} \times 20 \mu \mathrm{m},(\mathrm{C}) 10$ $\mu \mathrm{m} \times 10 \mu \mathrm{m},(\mathrm{M}-\mathrm{A})$ magnified region of $\mathrm{A},(\mathrm{M}-\mathrm{B})$ magnified region of $\mathrm{B}$.

As we well known, the $\beta$-form crystal has all trans $(\mathrm{T})$ conformation, whereas the $\alpha$ form crystal has trans $(\mathrm{T})$-gauche $(\mathrm{G})$-trans(T)-gauche' $\left(\mathrm{G}^{\prime}\right)$ (TGTG') conformation and the $\gamma$ phase has T3GT3G' conformation[13,14]. In addition, the evaporation-induced outward capillary flow can induce shear deformation of polymer coils. Therefore, PVDF $\beta$-form nucleation may be promoted by rich trans conformers which is caused by outward capillary flow induced extended polymer chains. If the time of reaching saturated concentration is shorter, the $\beta$-form nuclei should be dominated by rich trans conformers. Whereas, if the time of reaching saturated concentration is longer, the $\alpha$-form nuclei should be dominated because the gauche rich conformer rich state extended chains can be relaxed to back random coil state before crystallization and the $\alpha$-form phase nucleation is kinetically favorable process.

\subsection{Solvent evaporation induced crystal nucleation and morphology of PVDF/DMF solution small size droplet}

The diameter of dried PVDF/DMF solution S-droplet is about $\sim 80 \mu \mathrm{m}$ of diameter as shown in Figure 4 (A). If assuming the volume of droplet is mainly determined on the diameter of dried droplet and the evaporation rate is dependent on volume of droplet, the solvent evaporation time in the S-droplet should be about $10^{4}$ times fast than that in Ldroplet for same concentration of solution when the area ratio of dried small (with about $80 \mu \mathrm{m}$ of diameter) and larger (with about $5.02 \mathrm{~mm}$ of diameter) droplets is about $2.53 \times 10^{-}$ 4. As shown in Figure 1 and 4, the "coffee-ring" effect and morphology of dried PVDF/DMF solution S-droplet are different with that of dried PVDF/DMF solution Ldroplet. The "coffee ring" effect in dried PVDF/DMF solution S-droplet is showed more significant than that in dried PVDF/DMF solution L-droplet. As the size of droplet decreases, the thermal gradient becomes smaller. Accordingly, the Marangoni flow becomes negligible, and circular evaporation-induced flow transforms into an outward flow[2]. On the other hand, the morphology of dried PVDF/DMF solution S-droplet at edge and center regions are showed in Figure 4 (D) and (E), respectively. Aggregated particle-like crystals are presented as clusters in the edge region of dried PVDF/DMF solution S-droplet as shown in Figure 4 (D). In addition, the crystals in dried PVDF/DMF solution S-droplet 
have about more than hundred nanometers of size which are much smaller than that in dried PVDF/DMF solution L-droplet.

The crystal morphology of PVDF/DMF solution S-droplet in the edge and center regions are presented as particle-like crystals aggregated and grain-like crystals as shown in Figure $4(\mathrm{E})$. Based on the shape of crystals, we can judge that the $\alpha$-form crystals are almost absence in the dried PVDF/DMF solution S-droplet. Our previous work showed that the ultrasonic spray coated PVDF film was almost absence $\alpha$-phase[10]

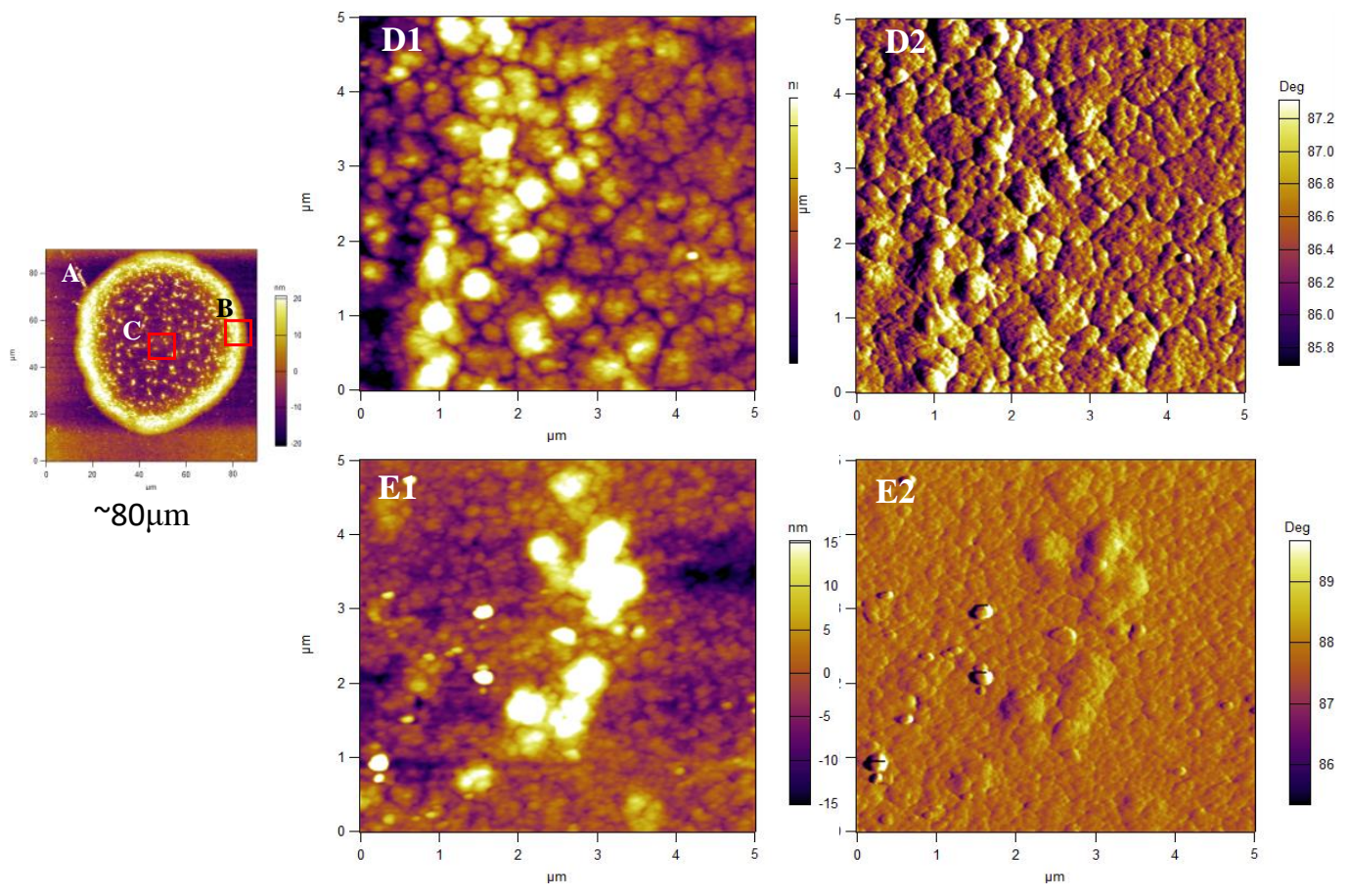

Figure 4. (A) AFM height image of dried PVDF/DMF solution droplet. (B) edge and (C) center regions of dried PVDF/DMF solution S-droplet. (D) and (E) AFM images of edge and center regions in dried PVDF/DMF solution S-droplet, respectively. (1) height image, (2) phase images, (A) $90 \mu \mathrm{m} \times 90 \mu \mathrm{m}$; (D) $5 \mu \mathrm{m} \times 5 \mu \mathrm{m}$; (E) $5 \mu \mathrm{m} \times 5 \mu \mathrm{m}$.

\subsection{Effect of evaporation rate on "coffee-ring" formation of dried small PVDF solution small size droplets}

To understand the effect of evaporation rate on outward capillary flow induced "coffee-ring" formation, we regulated the evaporation rate of solvent via increase of temperature and mixing with low boiling temperature of solvent. Since the boiling temperature of DMF and acetone is 153 and $56{ }^{\circ} \mathrm{C}$, respectively, the evaporation time of DMF/AC $(6 / 4$ $\mathrm{v} / \mathrm{v}$ ) mixture solvent at RT is shorter than that of DMF at RT. Therefore, the order of evaporation rate of solvents in S-droplets is increased as DMF at room temperature (RT) < $\mathrm{DMF} / \mathrm{AC}$ at $\mathrm{RT}<\mathrm{DMF}$ at $70{ }^{\circ} \mathrm{C}$. Figure 6 (A) and (B) show the AFM height images of dried PVDF/DMF solution S-droplets evaporated at RT and $70{ }^{\circ} \mathrm{C}$, respectively. And, the Figure 6 (C) shows the AFM height image of dried PVDF/DMF/AC solution S-droplet evaporated at RT. As shown in Figure 6 (A)-(C), the crystal distribution of dried PVDF solution Sdroplets at center region is dependent on evaporation rate. Among them, the crystals in center region of PVDF/DMF solution S-droplet dried at RT showed sparse distribution, whereas the crystals in the center region of PVDF/DMF solution S-droplet dried at $70{ }^{\circ} \mathrm{C}$ showed dense distribution as shown in Figure 6 (D). It indicates that the "coffee-ring" formation of dried PVDF solution S-droplets are dependent not only on evaporation rate but also on crystallization of PVDF during evaporation. 
Why fast enough solvent evaporation rate can unexpectedly reduce the "coffee-ring" formation in the case of dried PVDF solution S-droplets? When the concentration of polymer solution is reached to the saturated concentration, the crystal nucleation is occurred by the concentration excess (supersaturation) of the solution above the equilibrium (saturation). The time of reaching saturation concentration in the PVDF solution S-droplets is become to be shorter as increase of the solvent evaporation rate. And, the polymer chains in the dilute polymer solution S-droplets can be migrated to be edge of droplet or can be deformed by evaporation-induced outward capillary flow. On the other hand, the migrated polymer chains further can be crystallized to form "coffee-ring" at edge of droplet. Accordingly, the migration of polymer chains is promoted by a certain fast solvent evaporation rate, however is limited when the evaporation rate is further increased due to solidification.
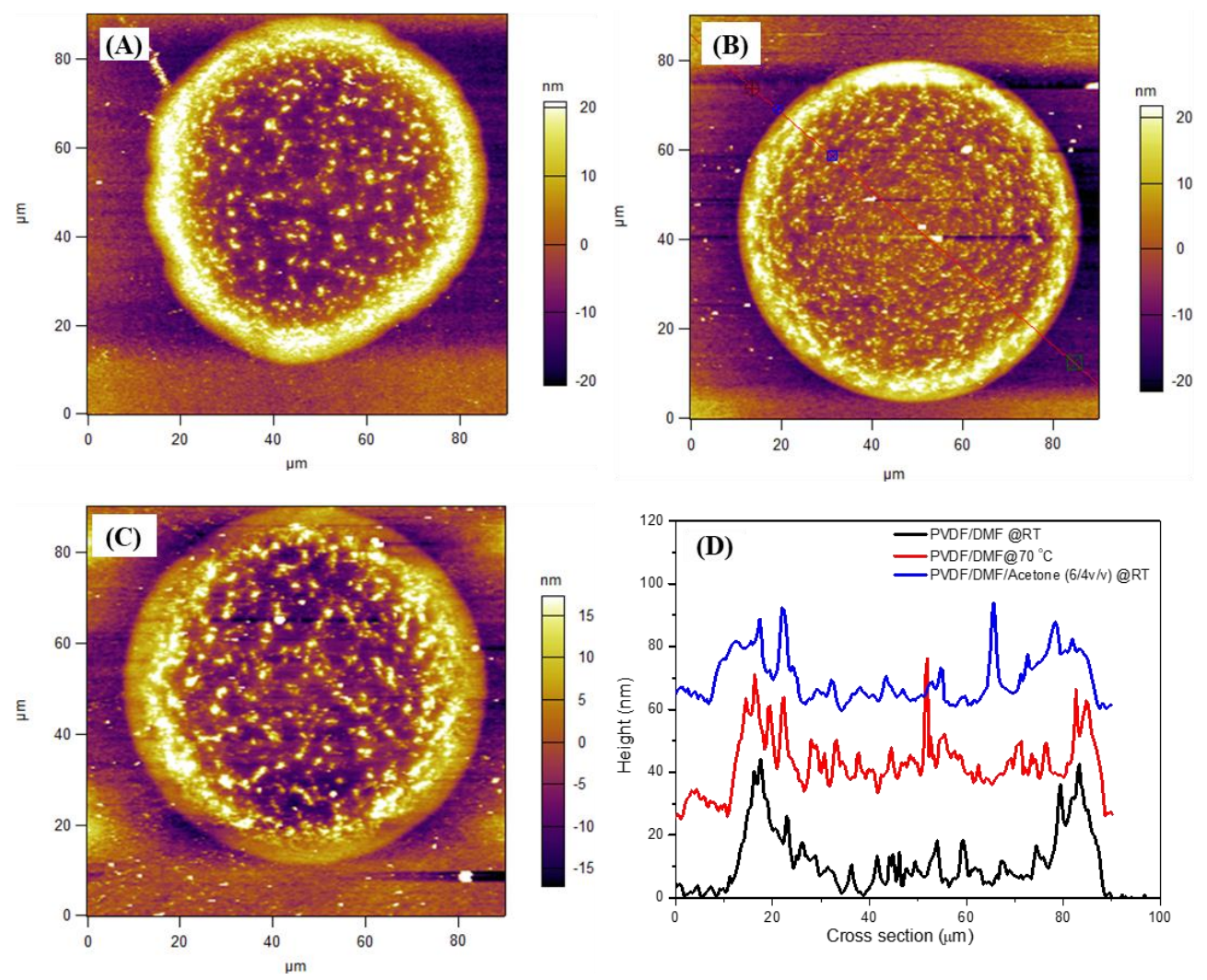

Figure 5. AFM height images of (A) PVDF/DMF solution S-droplets at RT, (B) PVDF/DMF solution S-droplets at $70{ }^{\circ} \mathrm{C},(\mathrm{C})$ PVDF/DMF/acetone solution S-droplets at RT, (D) cross section profiles obtained from AFM images of (A), (B) and (C).

\subsection{Effect of solution concentration on "coffee-ring" formation of dried PVDF/DMF/AC solution small-droplets}

To further understand the interplay between "coffee-ring" effect and crystallization, we investigated the effect of concentration on "coffee-ring" effect and crystallization patterns of dried PVDF/DMF/AC solution S-droplets. Figure 6 shows AFM images of dried $\mathrm{PVDF} / \mathrm{DMF} / \mathrm{AC}$ solution S-droplets with various concentration at RT. As shown in Figure 6, the "coffee-ring" effect of dried PVDF/DMF/AC solution S-droplets is weakened as increase of concentration. And, the crystal distribution in center region of dried PVDF/DMF/AC solution S-droplets is became to be denser and uniform as increase of concentration. For example, scattered distribution of polymer crystals is presented in the dried PVDF/DMF/AC solution S-droplet with $8 \mathrm{mg} / \mathrm{ml}$ of concentration, whereas, the dense distribution of polymer crystals is presented in the dried PVDF/DMF/AC solution S-droplet with $20 \mathrm{mg} / \mathrm{ml}$ of concentration as shown in Figure 7 (A) and (C). 
If the concentration is low (i.e., $8 \mathrm{mg} / \mathrm{ml}$ ), the time of reaching saturated concentration is become to be longer. Accordingly, the evaporation-induced outward capillary flow can promote the migration of polymer coils, and the deformation and aggregation of polymer chains are also enhanced before crystal nucleation. On the contrary, if the concentration is high, the time of reaching saturated concentration is become to be shorter. In this case, the migration of polymer chains should be restricted by crystal nucleation. Therefore, the "coffee-ring" effect is manly dominated by the competition between crystal nucleation and migration of polymer chains. On the other hand, the conformation and entanglement of polymer chains are strongly relative to concentration, which can also influence the migration of chains.

(A1)

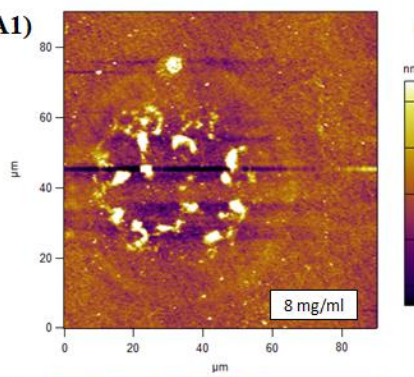

(A2) ${ }^{50}$

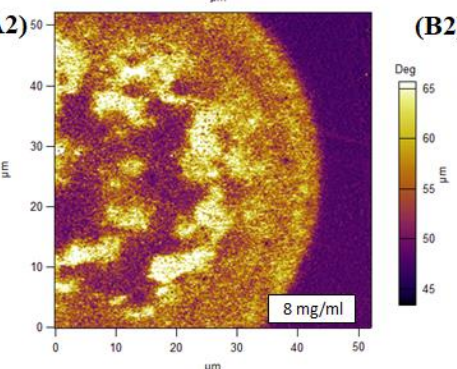

(B1)
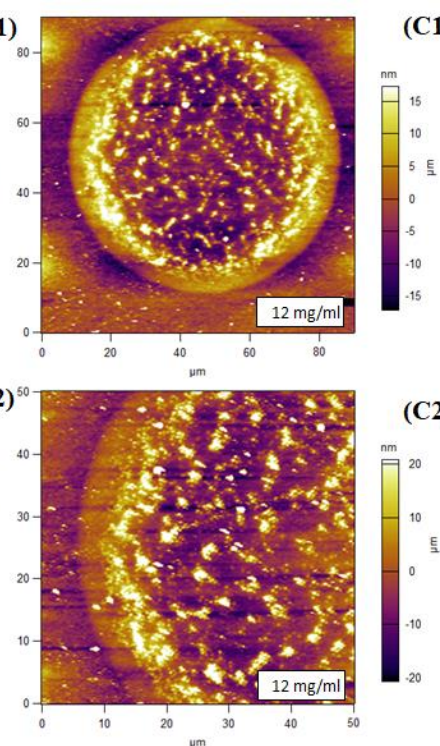

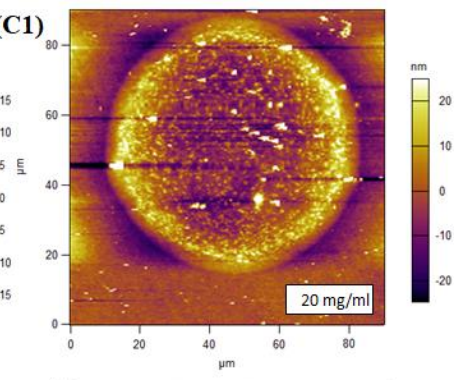

(C2)

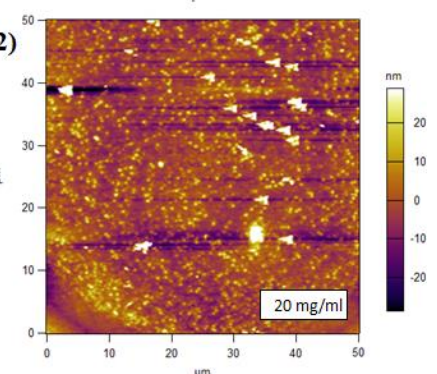

Figure 6. AFM (1) height and (2) phase images of PVDF/DMF/AC solution S-droplets with various concentrations. (A) $8 \mathrm{mg} / \mathrm{ml}$, (B) $12 \mathrm{mg} / \mathrm{ml}$, (C) $20 \mathrm{mg} / \mathrm{ml}$.

3.5. Effect of solution concentration on polymorphic and morphology of ultrasonic spray coated PVDF films

The solvent evaporation and droplets amalgamation may be simultaneously taken place when preparation of films by sprayed coating method. The crystal structure and morphology of spray coated PVDF films with various films were characterized by FTIR and AFM methods as shown in Figure 7 and 8, respectively. In Figure 7 (A), the bands at 614, 766, 795, 975 and $1402 \mathrm{~cm}^{-1}$ are sensitive to the $\alpha$-phase, and the bands at 840 and 1278 $\mathrm{cm}^{-1}$ are sensitive to the $\beta$-phase.[14] In addition, the band at $1234 \mathrm{~cm}^{-1} \mathrm{can}$ be assigned to $\gamma$-form phase.[14] As shown in Figure 7 (A), the band at 976, 763, and $612 \mathrm{~cm}^{-1}$ are evidently observed in the PVDF films except for the PVDF film with $12 \mathrm{mg} / \mathrm{ml}$ of concentration. It means that the $\alpha$-form phase is existed in the films except for the PVDF film with $12 \mathrm{mg} / \mathrm{ml}$ of concentration. 

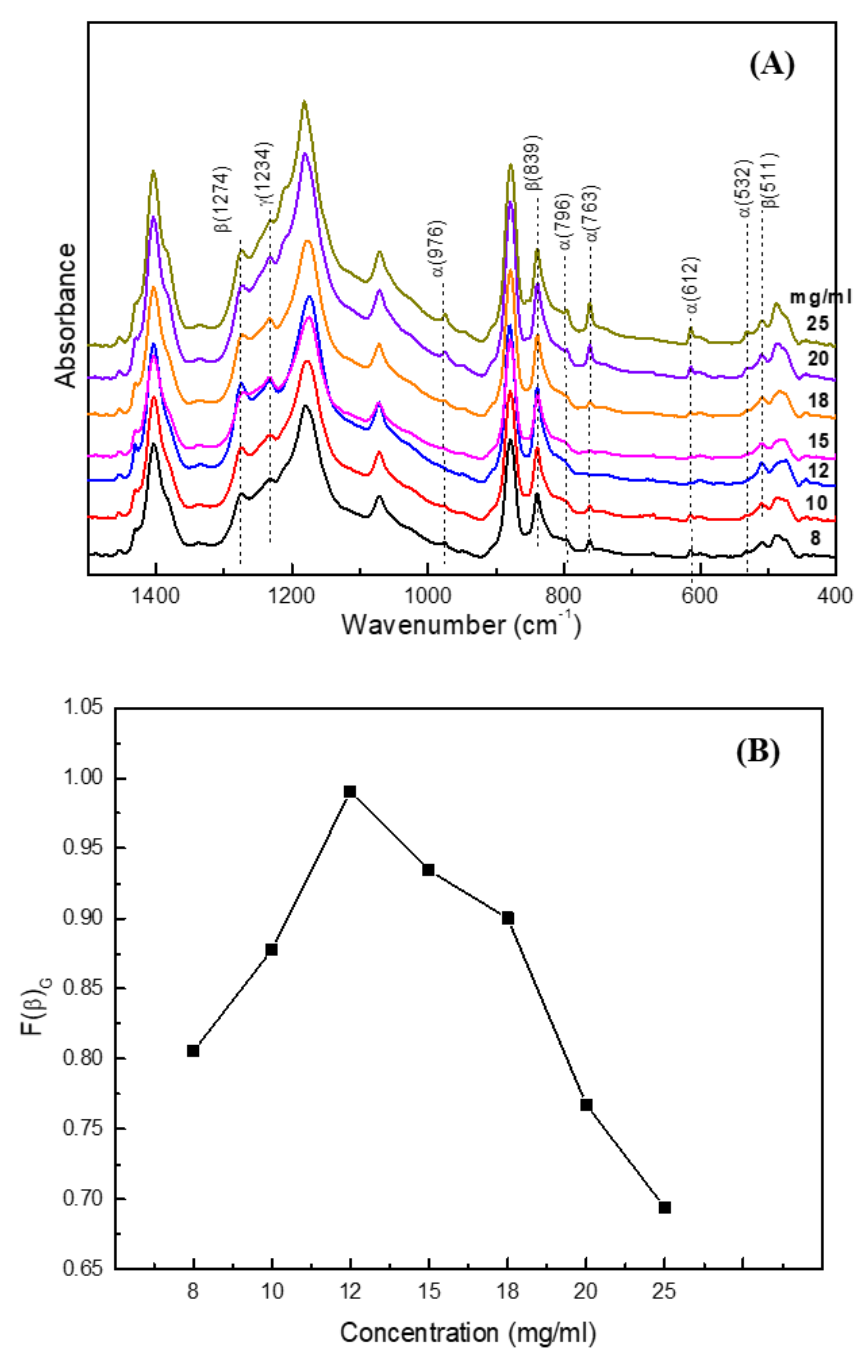

Figure 7. (A) FTIR spectra of ultrasonic spray coated PVDF films and (B) the solution concentration dependent relative fraction of $\beta$-form crystal $\left(\mathrm{F}(\beta)_{\mathrm{G}}\right)$.

The relative ratio of $\alpha$ - and $\beta$-phases can be estimated by the absorbances of characteristic bands of $\alpha$-and $\beta$-phases. Gregorio et al.[15] suggested that the relative $\beta$-phase content $\left(\mathrm{F}(\beta)_{\mathrm{G}}\right)$ can be determined by $\mathrm{F}(\beta)_{G}=\frac{A_{840}}{\left(\frac{K_{840}}{K_{766}}\right) A_{766}+A_{840}}$, where the $\mathrm{A}_{766}$ and $\mathrm{A}_{840}$ refer to the absorbance at 766 and $840 \mathrm{~cm}^{-1}$; and the K $766\left(6.1 \times 10^{4} \mathrm{~cm}^{2} \mathrm{~mol}^{-1}\right)$ and $\mathrm{K}_{840} \quad(7.7$ $\times 10^{4} \mathrm{~cm}^{2} \mathrm{~mol}^{-1}$ ) are the absorption coefficients of $766 \mathrm{~cm}^{-1}$ and $840 \mathrm{~cm}^{-1}$, respectively. The 766 and $840 \mathrm{~cm}^{-1}$ are sensitive to $\alpha$ - and $\beta$-phases, respectively. However, some researchers argued that the absorbance peak at $840 \mathrm{~cm}^{-1}$ is attributed to both $\beta$ - and $\gamma$-phases. Benz et al. [16] suggested that the relative $\beta$-phase content can be calculated based on the absorbance of the bands at 762 and $1272 \mathrm{~cm}^{-1}$ as $\mathrm{F}(\beta)_{B}=\frac{A_{1272}}{\left(\frac{K_{1272}}{K_{762}}\right) A_{762}+A_{1272}}$, where the $\mathrm{A} 762$ and $A_{1272}$ are absorbance of the bands at 762 and $1272 \mathrm{~cm}^{-1}$, respectively; and the $\mathrm{K}_{762}(0.365 \mu \mathrm{m}-$ $\left.{ }^{1}\right)$ and $K_{1272}\left(0.14 \mu \mathrm{m}^{-1}\right)$ are absorption coefficients of the bands at 762 and $1272 \mathrm{~cm}^{-1}$, respectively. The 762 and $1272 \mathrm{~cm}^{-1}$ are sensitive to $\alpha$ - and $\beta$-phase, respectively. Our calculated results showed that the calculated relative fraction of $\beta$-phase by both methods is similar. Therefore, we adopted the Gregorio et al. suggested method to calculate the relative fraction of $\beta$-form phase in this work. Figure 8 (B) shows the $F(\beta)_{G}$ of spray coated PVDF films. The $F(\beta)_{G}$ of spray coated PVDF films is increased as increase of concentration 
up to $12 \mathrm{mg} / \mathrm{ml}$, and then is decreased as increase of concentration. For example, the $\mathrm{F}(\beta)_{\mathrm{G}}$ of PVDF films with 8,12 and $20 \mathrm{mg} / \mathrm{ml}$ of concentrations is $0.80,0.99$ and 0.69 , respectively.

Since the $\alpha$ - and $\beta$-phase crystals of PVDF have different crystal morphology, the present of PVDF $\alpha$ - phase crystals can be observed by AFM. Figure 8 shows the AFM amplitude and phase images of PVDF films with 8,12 and $20 \mathrm{mg} / \mathrm{ml}$ of concentrations. The spherulite crystal morphology of PVDF is attribute to $\alpha$-phase crystals, and the particle-like crystal morphology of PVDF is attribute to $\beta$-phase crystals. In contrast with PVDF film with $12 \mathrm{mg} / \mathrm{ml}$ of concentration, the some spherulite crystals are observed in the AFM images of PVDF films with 8 and $20 \mathrm{mg} / \mathrm{ml}$ of concentrations.
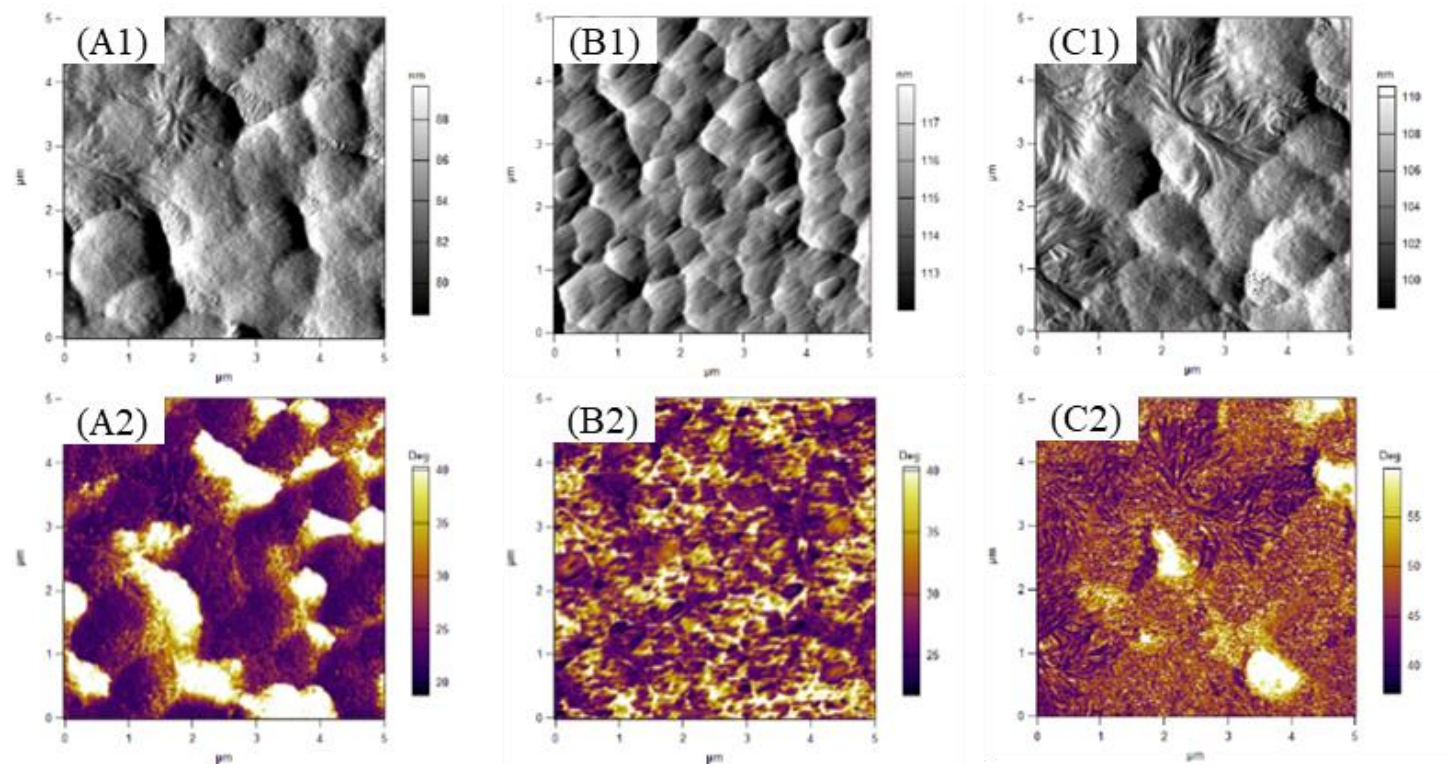

Figure 8. AFM (1) amplitude and (2) phase images of dried PVDF/DMF/acetone solution drop-lets with various concentrations. (A) $8 \mathrm{mg} / \mathrm{ml}$, (B) $12 \mathrm{mg} / \mathrm{ml}$, (C) $20 \mathrm{mg} / \mathrm{ml}$.

\section{Discussion}

It is generally recognized that the low evaporation rate is favorable to nucleation and growth of the thermodynamically stable $\beta$-form phase, whereas high evaporation rate yields the kinetically favorable metastable $\alpha$-form phase in the case of solvent casting. $[17,18]$ In addition, the PVDF $\beta$-phase is strongly dependent on solution type[19,20], temperature[15] and solvent evaporation rate[18] etc. For example, Gregorio[11] suggested that crystallization of PVDF from solution at temperature $\mathrm{T}<70{ }^{\circ} \mathrm{C}$ predominantly always results in the $\beta$-phase of PVDF, regardless of the solvent used, as long as it is a good solvent for PVDF. As temperature increases $\alpha$-phase formation increases, becoming predominant at $\mathrm{T}>110^{\circ} \mathrm{C}$. Ma et al.[21] investigated the effect of mixed solvents on the crystallization of PVDF. Their results showed that the crystallization of PVDF from tetrahydrofuran (THF)/N, N-dimethylformamide (DMF) mixed solvents yielded the $\alpha$ - and $\beta$ form phases, and the $\beta$-form phase content was proportional to the DMF content. Zhao et al. [22] also investigated the crystallization of PVDF from the DMF/ethanol mixed solvent nonsolvent. They found that low ethanol content favored the formation of $\beta$-form phase, while high ethanol content resulted predominantly in $\alpha$-form phase. Gregorio et al.[18] investigated the effect of solvent evaporation rate on the crystalline phase of solvent-cast PVDF films. They suggested that the low evaporation rate favors nucleation and growth of the thermodynamically stable $\beta$-form phase, whereas high evaporation rates yield the kinetically favorable metastable $\alpha$ phase. When the PVDF concentration increases during the process and growth takes place, there nuclei are already in the phase determined by the initial evaporation rate. They argued that the competition in the formation of the $\alpha$ and $\beta$-form phases does not occur during the whole crystallization process, as the distinct, constant evaporation rates during nucleation are important for leading to different 
relative amounts of the crystalline phases. Horibe et al.[17] also investigated the relationship between PVDF crystalline structure and solvent evaporation rate. They prepared spin-coated PVDF films with various solvents and the solvent evaporation rate was quantified by measuring the sample weight with an electronic balance. Their results indicated that the crystalline structures of forms I ( $\beta$-form), II ( $\alpha$-form) and III ( $\gamma$-form) of PVDF were obtained when the solvent evaporation rates were $<0.0001,>0.2$ and between 0.03 and $0.00058 \mathrm{~g} \mathrm{~min}^{-1}$, respectively. Recently, Nishiyama et al.[20] investigated the effect of the solvent evaporation rate and solvent type on the crystal formation of PVDF prepared by spin-coating method. Their results showed that the crystalline phase of PVDF changed in the order of $\alpha-, \gamma$ - and $\beta$-form with the increasing dipole moment of the solvent in the much-solvent-remaining state, and the crystalline structure of PVDF with a higher dipole moment of solvents (hexamethyl phosphor amide) was dominantly dependent on the evaporation rate in the almost all solvent evaporated state, and varied in the order of $\beta$-, $\gamma$ - and $\alpha$-form with the increasing solvent evaporation rate. However, PVDF with lower dipole moment of solvents (triethyl phosphate) always formed $\alpha$-form phase, regardless of the evaporation rate.

However, the crystallization of PVDF solution in the droplets is different with that in the casting or spin coating due to occurring evaporation induced flows. In the case of droplets, the migration and shear deformation of polymer chains generally occur during solvent evaporation. The solvent evaporation-induced outward capillary flow can induce migration of chains and the shear deformation of polymer chains to cause the conformational transition of gauche to trans. Therefore, the nucleation of PVDF $\beta$-form crystals is promoted by droplet evaporation at near boundary of droplet as shown in Figure 9. And, the crystal growth of PVDF is strongly restricted by the evaporation resulted solidification. The "coffee-ring" effect of dried PVDF droplets was mainly caused by accumulated crystal nucleation at edge region of droplet, which was enhanced by migrated polymer chains. The slow solidification of droplets can promote $\alpha$-form phase nucleation because the trans conformers can be transformed to gauche conformers by relaxation and the $\alpha$ form phase is kinetically favorable. The fast solvent evaporation can strongly restrict the crystal growth, results in forming sub-micrometer size of crystals. In addition, the formation of "coffee ring" in the S-droplets is strongly influenced by droplet size, solvent evaporation rate and concentration. Accordingly, the final crystal structure and morphology of dried PVDF S-droplets and spray coated PVDF films are determined by the interplay between evaporation-induced migration of polymer chains, crystallization, and solidification.

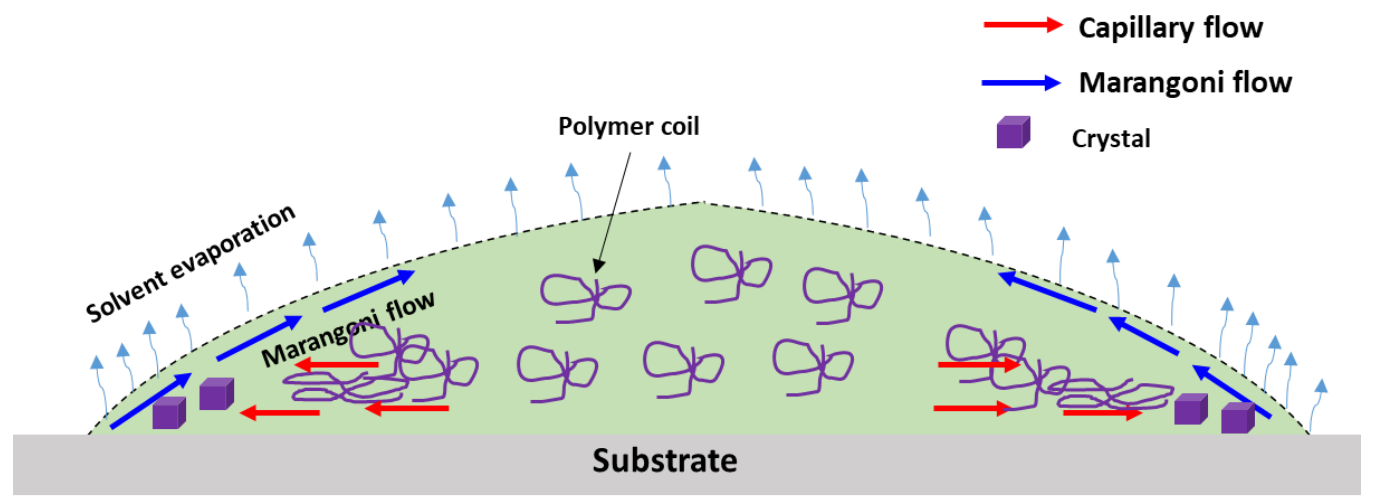

Figure 9. Schematic illustration of solvent evaporation induced flows and crystallization of PVDF droplet during solvent evaporation. 


\section{Conclusions}

In this work, we investigated the effect of droplet size, solvent evaporation rate and concentration on "coffee-ring" effect, crystal structure, and morphology of dried PVDF solution droplets. The crystal morphology and crystal distribution of dried PVDF droplets in the center and edge regions were different due to "coffee-ring" effect. The "coffee-ring" effect of dried PVDF droplets was mainly composited of accumulated crystals at the edge region of droplet, which was mainly made by crystallization of migrated chains. The "coffee-ring" effect of PVDF droplets was dependent on droplet size, solvent evaporation rate and concentration, which was determined by interplay between migration of chains and solidification. In addition, the nucleation of PVDF $\beta$-form phase was promoted by fast solvent evaporation induced outward capillary flow and the crystal growth was significantly restricted by fast solidification in the case of droplet evaporation. Our results showed that the sub-millimeter size droplets can be obtained by ultrasonic spray coating method, and the decrease of droplet size and controlling the solvent evaporation were effective ways to improve the $\beta$-phase content and decrease the crystal size of PVDF.

Author Contributions: Conceptualization, writing - review and editing, project administration, and funding acquisition, Y. Liang; and validation, data curation, investigation, original draft preparation, S. Wang. All authors have read and agreed to the published version of the manuscript.

Funding: This research was funded by Natural Science Foundation of Beijing Municipality, China, grant number 2172023.

Conflicts of Interest: The authors declare no conflict of interest.

\section{References}

1. Diao, Y.; Shaw, L.; Bao, Z.; Mannsfeld, S.C.B. Morphology control strategies for solution-processed organic semiconductor thin films. Energy Environ. Sci. 2014, 7, 2145-2159, doi:10.1039/c4ee00688g.

2. Zang, D.; Tarafdar, S.; Tarasevich, Y.Y.; Dutta Choudhury, M.; Dutta, T. Evaporation of a Droplet: From physics to applications. Physics Reports 2019, 804, 1-56, doi:10.1016/j.physrep.2019.01.008.

3. Deegan, R.D.; Bakajin, O.; Dupont, T.F.; Huber, G.; Nagel, S.R.; Witten, T.A. Capillaryflowasthecause of ring stains fromdried liquid drops. Nature 1997, 389, 827-829.

4. Zhou, J.; Man, X.; Jiang, Y.; Doi, M. Structure Formation in Soft-Matter Solutions Induced by Solvent Evaporation. Advanced Materials 2017, 29, 1703769, doi:10.1002/adma.201703769.

5. Deegan, R.D.; Bakajin, O.; Dupont, T.F.; Huber, G.; Nagel, S.R.; Witten, T.A. Contact line deposits in an evaporating drop. Phys Rev E 2000, 62, 756-765.

6. Yu, X.; Xing, R.; Peng, Z.; Lin, Y.; Du, Z.; Ding, J.; Wang, L.; Han, Y. To inhibit coffee ring effect in inkjet printing of lightemitting polymer films by decreasing capillary force. Chinese Chemical Letters 2019, 30, 135-138, doi:10.1016/j.cclet.2018.09.007.

7. Mampallil, D.; Eral, H.B. A review on suppression and utilization of the coffee-ring effect. Adv Colloid Interface Sci 2018, 252, 38-54, doi:10.1016/j.cis.2017.12.008.

8. Hu, Y.; Zhang, X.; Qiu, M.; Wei, Y.; Zhou, Q.; Huang, D. From coffee ring to spherulites ring of poly(ethylene oxide) film from drying droplet. Appl Surf Sci 2018, 434, 626-632, doi:10.1016/j.apsusc.2017.10.202.

9. Rietveld, I.B.; Kobayashi, K.; Yamada, H.; K.Matsushige. Morphology control of poly(vinylidene fluoride) thin film made with electrospray. Journal of Colloid and Interface Science 2006, 298, 639-651.

10. Wang, S.; Liang, Y. Transparent and ferroelectric poly (vinylidene fluoride) film achieved by simple ultrasonic spray coating method. Materials Letters 2019, 247, 71-74, doi:10.1016/j.matlet.2019.03.096.

11. Gregorio, R. Determination of the $\alpha, \beta$, and $\gamma$ crystalline phases of poly(vinylidene fluoride) films prepared at different conditions. J Appl Polym Sci 2006, 100, 3272-3279, doi:10.1002/app.23137. 
12. Kang, S.J.; Park, Y.J.; Sung, J.; Jo, P.S.; Park, C.; Kim, K.J.; Cho, B.O. Spin cast ferroelectric beta poly(vinylidene fluoride) thin films via rapid thermal annealing. Appl Phys Lett 2008, 92, 012921, doi:10.1063/1.2830701.

13. Lovinger, A.J. Ferroelectric Polymers. Science 1983, 220, 1115-1121.

14. Martins, P.; Lopes, A.C.; Lanceros-Mendez, S. Electroactive phases of poly(vinylidene fluoride): Determination, processing and applications. Progress in Polymer Science 2014, 39, 683-706, doi:10.1016/j.progpolymsci.2013.07.006.

15. Rinaldo Gregorio, J.; Cestari, M. Effect of crystallization temperature on the crystalline phase content and morphology of poly(vinylidene fluoride). Journal of Polymer Science: Part B: Polymer Physics 1994, 32, 859-870, doi:10.1002/polb.1994.090320509.

16. Benz, M.; Euler, W.B. Determination of the Crystalline Phases of Poly(vinylidene fluoride) Under Different Preparation Conditions Using Differential Scanning Calorimetry and Infrared Spectroscopy. J Appl Polym Sci 2003, 89, $1093-1100$.

17. Horibe, H.; Sasaki, Y.; Oshiro, H.; Hosokawa, Y.; Kono, A.; Takahashi, S.; Nishiyama, T. Quantification of the solvent evaporation rate during the production of three PVDF crystalline structure types by solvent casting. Polym J 2004, 46, 104110.

18. Chinaglia, D.L.; Jr., R.G.; Stefanello, J.C.; Altafim, R.A.P.; Wirges, W.; Wang, F.; Gerhard, R. Influence of the solvent evaporation rate on the crystalline phases of solution-cast poly(vinylidene fluoride) films. J Appl Polym Sci 2010, 116, doi:10.1002/app.31488.

19. Park, Y.J.; Kang, Y.S.; Park, C. Micropatterning of semicrystalline poly(vinylidene fluoride) (PVDF) solutions. Eur Polym J 2005, 41, 1002-1012.

20. Nishiyama, T.; Sumihara, T.; Sato, E.; Horibe, H. Effect of solvents on the crystal formation of poly (vinylidene fluoride) film prepared by a spin-coating process. Polym J 2016, 1-7.

21. Ma, W.; Zhang, J.; Chen, S.; Wang, X. Crystalline Phase Formation of Poly(vinylidene fluoride) from Tetrahydrofuran/N,N dimethylformamide Mixed Solutions. Journal of Macromolecular Science, Part B: Physics 2008, 47, $434-449$.

22. Zhao, X.; Cheng, J.; Chen, S.; Zhang, J.; Wang, X. Controlled Crystallization of Poly(vinylidene fluoride) Chains from Mixed Solvents Composed of Its Good Solvent and Nonsolvent. Journal of Polymer Science: Part B: Polymer Physics 2010, 48, 575-581. 